\title{
OPTIMIZATION OF THE USE OF CARBON PASTE ELECTRODES (CPE) FOR ELECTROCHEMICAL STUDY OF THE CHALCOPYRITE
}

\author{
Daniela G. Horta, Denise Bevilaqua, Heloísa A. Acciari, Oswaldo Garcia Júnior* e Assis V. Benedetti \\ Instituto de Química, Universidade Estadual Paulista, CP 355, 14801-970 Araraquara - SP, Brasil
}

Recebido em 30/6/08; aceito em 2/3/09; publicado na web em 4/8/09

\begin{abstract}
The use of carbon paste electrodes (CPE) of mineral sulfides can be useful for electrochemical studies to overcome problems by using massive ones. Using CPE-chalcopyrite some variables were electrochemically evaluated. These variables were: (i) the atmosphere of preparation (air or argon) of CPE and elapsed time till its use; (ii) scan rate for voltammetric measurements and (iii) chalcopyrite concentration in the CPE. Based on cyclic voltammetry, open-circuit potential and electrochemical impedance results the recommendations are: oxygen-free atmosphere to prepare and kept the CPE until around two ours, scan rates from 10 to $40 \mathrm{mV} \mathrm{s}^{-1}$, and chalcopyrite concentrations $\geq 20 \%$.
\end{abstract}

Keywords: carbon paste electrodes (CPE); chalcopyrite; leaching.

\section{INTRODUCTION}

The mechanisms of mineral sulphides dissolution have been investigated mainly for copper minerals, in order to improve the efficiency of the industrial leaching operations, including bacterial processes. The oxidation of sulphides by a chemical or biological oxidant can be look upon as an electrochemical reaction and it can be consequently studied by electrochemical techniques.

Traditional electrochemical techniques including cyclic voltammetry (CV) and electrochemical impedance spectroscopy (EIS) have been utilized in these studies with mineral sulphides with or without bacterial involvement. ${ }^{1-4}$ These methods permit to study electrical interactions between the electrode and the electrolyte, and how these interactions change with different oxidation potentials.

Among the metal sulphides that are oxidized by bacterial or chemical attack, chalcopyrite $\left(\mathrm{CuFeS}_{2}\right)$ has deserved special attention since it is the principal mineral source of the ten million tons of copper annually produced worldwide..$^{5-7}$

In all these studies the electrodes are prepared, in general, with small blocks of natural sulphides. However, some critical problems during electrode preparation, such as, tendency to fracture in the polishing procedure and irregular and heterogeneous surface, can affect the confidence of data recorded during experiment time course.

To overcome these problems the use of carbon paste electrode (CPE), which is prepared with powdered mineral sulphides, has been investigated by some authors. ${ }^{8-11}$ However, a more specific work to evaluate the repeatability of these CPE has no precedent in the literature.

The CPE is composed by a paste made from graphite powder, binding material, and pulverized mineral. The granulation of the graphite and the powder mineral needs to be the same to avoid physical problems and heterogeneity. For homogenization a volatile organic substance that does not alter the chemical and physical properties of the paste is usually used.

The purpose of this work was to evaluate the electrochemical response of CPE-chalcopyrite in an acidic medium using electrochemical techniques, in order to validate the use of this electrode in electrochemical studies. It was investigate the repeatability of the CPE packing regarding the atmosphere of preparation and storage

\footnotetext{
*e-mail: oswaldo@iq.unesp.br
}

(air and argon), influence of scan rate in cyclic voltammetry (CV) measurements and chalcopyrite concentration in the CPE.

\section{EXPERIMENTAL}

Chalcopyrite $\left(\mathrm{CuFeS}_{2}\right)$ from Sossego mine, state of Pará, Brazil, was obtained from Vale Do Rio Doce Company. The mineral was ground in a disc mill to $100 \%<37 \mu \mathrm{m}$ (400 mesh).

A total of $0.4 \mathrm{~g}$ of graphite (Aldrich), $0.1 \mathrm{~g}$ of chalcopyrite $(<400$ mesh) and $0.075 \mathrm{~g}$ of nujol (mineral oil) was mixed with chloroform to obtain a homogenous paste which was preserved under oxygen-free atmosphere. This paste was placed in a cavity on the electrode body, in contact with a platinum disk of geometric area of $7.5 \mathrm{~mm}^{2}$.

All the solutions were prepared with analytical grade reagents and Milli-Q water, resistivity $18 \mathrm{M} \Omega \mathrm{cm}$. As electrolytic solution it was used $\mathrm{T} \& \mathrm{~K}$ medium $^{12}$ without ferrous ions with the following composition: $0.5 \mathrm{~g}$ of $\left(\mathrm{NH}_{4}\right)_{2} \mathrm{SO}_{4}, 0.5 \mathrm{~g}$ of $\mathrm{K}_{2} \mathrm{HPO}_{4}$ and $0.5 \mathrm{~g}$ of $\mathrm{MgSO}_{4} 7 \mathrm{H}_{2} \mathrm{O}$ in purified water (q.s.p. $800 \mathrm{~mL}$ ), $\mathrm{pH} 1.8$ adjusted with $\mathrm{H}_{2} \mathrm{SO}_{4}$, which has been used as a nutrient medium to cultivate $A$. ferrooxidans. In the $\mathrm{CV}$ experiments $0.1 \mathrm{~mol} \mathrm{~L}^{-1} \mathrm{~K}_{2} \mathrm{SO}_{4}$ was added as supporting electrolyte.

For electrochemical experiments an $\mathrm{Ag}|\mathrm{AgCl}| \mathrm{KCl}_{\text {sat }}$ connected to the solution through a Luggin capillary was used as reference electrode. A platinum wire was the auxiliary electrode and the CPEchalcopyrite the working one.

Four different conditions were evaluated using cyclic voltammetry: the influence of atmosphere of the paste preparation (air or argon atmosphere); the elapsed preparation time using the same lot of paste; the influence of scan rate $(v)$ and, the influence of the chalcopyrite percentage in the paste. For each cyclic voltammogram the paste was removed from the electrode and a new quantity of paste of the same lot was used. Mean values, standard deviation, confidence interval and scatter diagrams of the voltammograms obtained were used for statistical analysis.

The influence type of atmosphere and elapsed preparation time was evaluated by successive cyclic voltammograms at $10 \mathrm{mV} \mathrm{s}^{-1}$ with CPE-(20 wt.\%) chalcopyrite. The influence of the scan rate was investigated by recording cyclic voltammograms of CPE-(20 wt.\%) chalcopyrite electrodes at $5 \leq \mathrm{v} \leq 50 \mathrm{mV} \mathrm{s}^{-1}$ using paste prepared and kept in argon. A minimum of 5 repetitions was carried out for each scan rate and the paste was changed after each cyclic voltam- 
mogram. Therefore, for analyzing the electrochemical results each cyclic voltammogram was supposed to be independent. For these studies four lots of paste were prepared. The first one was utilized to obtain all $\mathrm{CVs}$ at 5, 10 and $20 \mathrm{mV} \mathrm{s}^{-1}$. Second, third and fourth lots were used to record cyclic volammograms at 30,40 and $50 \mathrm{mV}$ $\mathrm{s}^{-1}$, respectively.

For evaluation of chalcopyrite percentage in the paste cyclic voltammetry, open-circuit potential (OCP) measurements and electrochemical impedance spectroscopy were used. The cyclic voltammograms were recorded at $20 \mathrm{mV} \mathrm{s}^{-1}$ and using the following different chalcopyrite concentrations: $2.5 ; 5 ; 10 ; 20 ; 30 ; 40 ; 50$ and $60 \%(\mathrm{w} / \mathrm{w})$. The impedance spectra were recorded for the same concentrations, except $2.5 \%$. EIS measurements were done in triplicate at $25{ }^{\circ} \mathrm{C}$, with the past prepared and kept in argon atmosphere. The impedance diagrams were recorded only after the steady state potential has been reached.

The cyclic voltammograms were obtained using MQPG-01 potentiostat coupled to a computer by means of a MQI12/8PCC interface. Open-circuit potential (OCP) and electrochemical impedance (EIS) measurements of the CPE-chalcopyrite were performed using an EG\&G PARC potentiostat, model 283 and EG\&G PARC, model 1025 frequency response analyzer monitored by a microcomputer using the M398 software.

EIS measurements were performed using a conventional threeelectrode electrochemical cell with a CPE-chalcopyrite as working electrode. A Pt disc electrode connected to the reference by a $10 \mathrm{uF}$ capacitor was used to minimize the noise at low frequency range and the phase shift at high frequency region. ${ }^{13} \mathrm{An} \mathrm{Ag}|\mathrm{AgCl}| \mathrm{KCl}_{\text {sat }}$ was used as reference electrode. The impedance spectra were obtained by applying on the OCP value a small amplitude potential wave (10 $\mathrm{mV} \mathrm{rms}$ ). The frequency range was varied from $20 \mathrm{kHz}$ to $10 \mathrm{mHz}$ recording 10 points per decade.

\section{RESULTS AND DISCUSSION}

\section{Effect of the paste storage and the scan rate $(v)$}

Figure 1 illustrates 12 replicates of cyclic voltammograms successively recorded for CPE-(20 wt.\%) chalcopyrite prepared and kept in an air atmosphere. These cyclic voltammograms showed only one anodic current peak centered at around $+1 \mathrm{~V}$ and no cathodic current peak was observed up to $-0.2 \mathrm{~V}$, suggesting that the products of chalcopyrite oxidation are not reduced in the potential interval from +1.5 to $-0.2 \mathrm{~V}$.

There are different propositions concerning mineral oxidation: the formation of covellite (CuS) or non-stoichiometric metal sulfides,

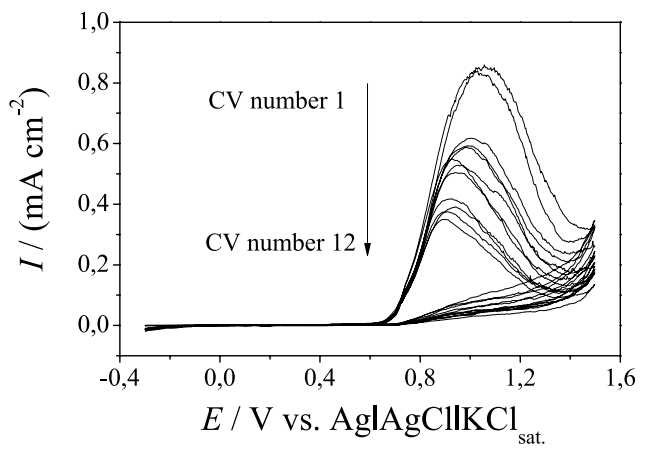

Figure 1. Twelve replicate voltammograms obtained for CPE-(20 wt.\%) chalcopyrite in $T \& K$ medium $+0.1 \mathrm{~mol} \mathrm{~L}^{-1}$ of $K_{2} \mathrm{SO}_{4}$ at $\mathrm{V}=10 \mathrm{mV} \mathrm{s}^{-1}$, initiating the scan in the anodic direction. The paste was prepared and stored in an air atmosphere sulfur, and copper (II) and iron (III/II) soluble ions. ${ }^{14-17}$ Recently, it was proposed the previous formation of non-stoichiometric sulfides on the chalcopyrite surface according to Equation $1 .{ }^{18-20}$

$\mathrm{CuFeS}_{2} \rightarrow x \mathrm{Cu}^{2+}+y \mathrm{Fe}^{2+}+\mathrm{Cu}_{1-\mathrm{x}} \mathrm{Fe}_{1-\mathrm{y}} \mathrm{S}_{2}+2(x+y) \mathrm{e}^{-}$

The action of oxygen from the atmosphere on the mineral surface was not considered in Equation 1. As mentioned above, the maximum observed at around $1.0 \mathrm{~V}$ can be attributed to the chalcopyrite oxidation, probably to form non-stoichiometric metal sulfides and $\mathrm{Cu}^{2+}$ and $\mathrm{Fe}^{3+/ 2+}$ ions in solution, since the formal potential of the $\mathrm{Fe}^{3+/ 2+}$ redox couple is around $+0.6 \mathrm{~V}^{21}$ However the iron ions concentration is probably very low since no cathodic peak associated with its reduction was detected.

The main changes observed in the successive CVs of Figure 1 with elapsed time are the decrease in the anodic current and a slight shifting of peak potential towards less positive values. The decrease in the current peak was interpreted as consequence of the ageing of the paste in presence of air which leads to chalcopyrite oxidation. After the second replicate (around $10 \mathrm{~min}$ ) there is no more repeatability. It is well-know that chalcopyrite undergoes spontaneous oxidation ${ }^{22}$ which could lead to form oxide films. The displacing of the peak potential to less positive values suggests that the modified surface is easier oxidized, while the formation of a passive film is expected to increase the oxidation potential. This result cannot invalidate the possibility to occur spontaneous oxidation of the mineral by air to form iron and copper oxides. Probably, a heterogeneous oxide film is formed, which presents non typical passive behavior, but influences the CV curves decreasing the current peaks. However, the shifting potential towards less positive values will need to be explored in future experimental work.

By using CPE-(20 wt.\%) chalcopyrite prepared and kept under argon atmosphere it was detected a remarkable repeatability of four replicates of cyclic voltammograms recorded successively at $10 \mathrm{mV}$ $\mathrm{s}^{-1}$. So, another experiment was carried out with the same procedure of CPE preparation in which the elapsed time was controlled until the cyclic voltammograms were recorded (Figure 2). This figure shows no considerable effect on anodic charge in approximately two hours after the paste preparation. These CPE-chalcopyrite electrodes showed much better repeatability compared to that obtained with a paste prepared in kept in air.

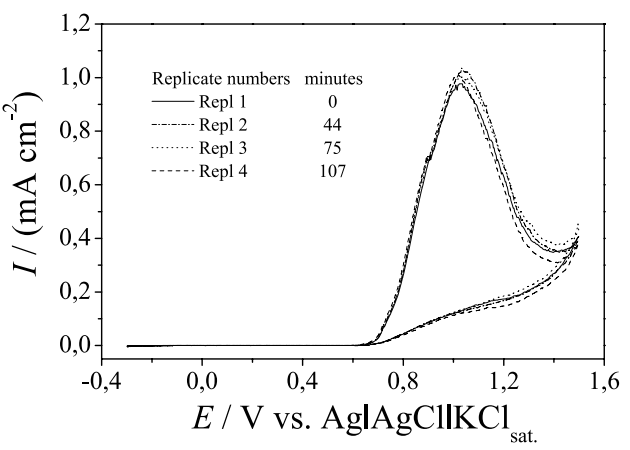

Figure 2. Four replicate voltammograms obtained for CPE-(20 wt.\%)chalcopyrite in $T \& K$ medium $+0.1 \mathrm{~mol} \mathrm{~L}^{-1}$ of $\mathrm{K}_{2} \mathrm{SO}_{4}$ at $\mathrm{v}=10 \mathrm{mV} \mathrm{s}^{-1}$, initiating the scan in the anodic direction. The paste was prepared and stored in an argon atmosphere

A statistical analysis was performed in order to compare the parameters extracted from all cyclic voltammograms. In the presence and absence of oxygen, the anodic charge values were used for the statistical analysis because this parameter is more representative 


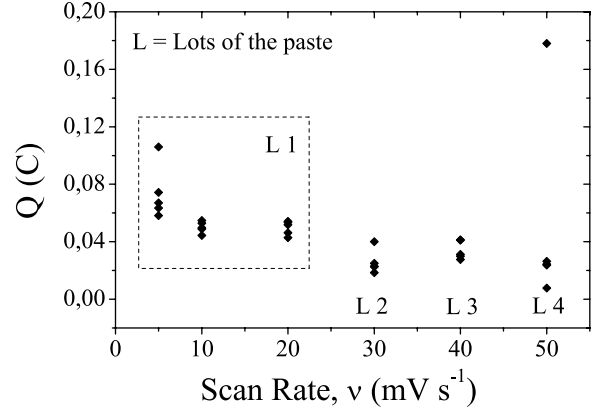

Figure 3. Scattering diagram: data for five replicate voltammograms for each scan rate corresponding to the CPE-(20 wt. \%)chalcopyrite in $T \& K$ medium $+0.1 \mathrm{~mol} \mathrm{~L}^{-1}$ of $\mathrm{K}_{2} \mathrm{SO}_{4}$

of the I-E curves. As the cyclic voltammograms were considered independent a normal distribution could be supposed for these results. Student's t-test was applied on the average of charge values and the $p$ value was 0.025 and showed that these charges are quite different at confidence interval of $95 \%$. The standard deviations of the anodic charges of the paste kept in air and argon atmospheres were 0.01 and 0.001 , respectively. Therefore the magnitude of the deviation obtained using the paste under argon atmosphere was about 10 times lower than in air. These results also demonstrate the spontaneous oxidation of chalcopyrite powder present in the paste prepared in air.

Definitely the paste kept under argon atmosphere must be recommended to be used for further experiments, since it prevents chalcopyrite oxidation.

To study the influence of the scan rate five replicates of $\mathrm{CV}$ s were performed at the following rates: 5,10 and $20 \mathrm{mV} \mathrm{s}^{-1}$ (first lot of the paste) and 30,40 and $50 \mathrm{mV} \mathrm{s}^{-1}$ (second, third and fourth lots, respectively). Figure 3 shows an example of scatter diagram for the anodic charge values versus scan rate $(v)$, and contains all repetitions for each $v$ value.

The scatter diagrams allow to analyse data without to convert them to another format (calculate mean values) or make assumptions about what type of probability density function (statistical distribution) should be used to calculate mean values. ${ }^{23}$ Scatter diagrams were also useful to verify which scan rate among the values used showed better repeatability in relation to the type packing of carbon paste.

The charge values were almost constant independent on the elapsed time represented by the different points obtained at the same scan rate. For 5 and $50 \mathrm{mV} \mathrm{s}^{-1}$ some dispersion in the charge values was observed. The lowest dispersion was observed for scan rates 10 and $20 \mathrm{mV} \mathrm{s}^{-1}$ and then the last one was chosen for further measurements. It was also observed a little decrease in the charge for higher values of $v$ due to the decrease in the time of electrolysis. Differences in the thickness of paste wetted by the electrolyte may also contribute to decrease the anodic charge. For instance, at $5 \mathrm{mV} \mathrm{s}^{-1}$ the time contact with the electrolyte is higher and consequently, higher can be the thickness of the paste wetted. In general the repetitions can be considered satisfactory except for a value recorded at $50 \mathrm{mV} \mathrm{s}^{-1}$ $(0.18 \mathrm{C})$, which can be attributed to experimental error.

\section{EFFECT OF CHALCOPYRITE PERCENTAGE IN THE PASTE}

The influence of chalcopyrite concentration was evaluated using CV, OCP and EIS in order to identify the better chalcopyrite concentration in the paste that can be applied in the electrochemical studies involving this mineral. The pastes were prepared and kept in argon atmosphere.
Initially it was performed CV measurements in triplicate at 20 $\mathrm{mV} \mathrm{s}^{-1}$ between 0.5 and $1.8 \mathrm{~V}$ using CPE with different chalcopyrite concentrations. Figure 4A illustrates one I-E curve for each chalcopyrite concentration. It can be observed that anodic peak attributed to the oxidation of the mineral increases with the increase of the chalcopyrite concentration, and the peak potentials were shifted to more positive values. This displacement of peak potentials can be due to the increase in the semiconductor character of the electrode as increase the chalcopyrite concentration.
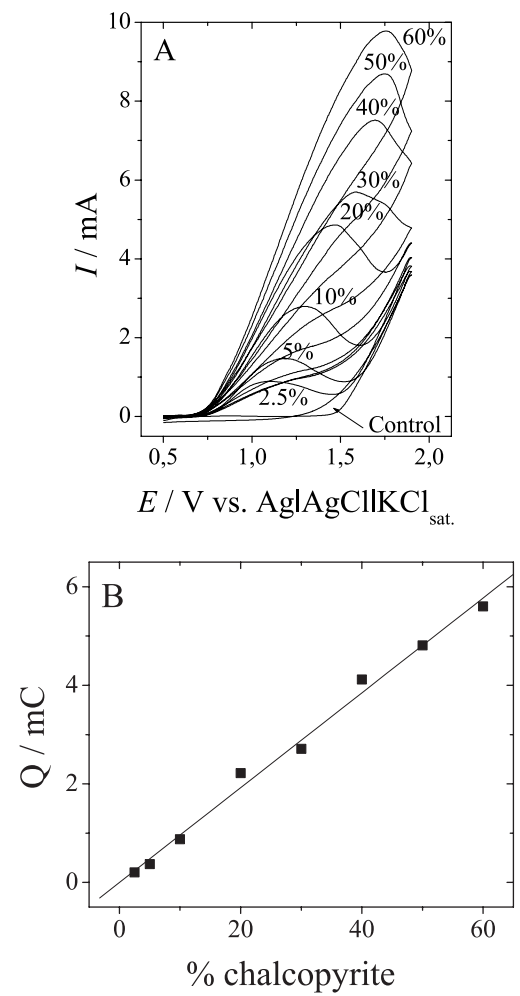

Figure 4. (A) Cyclic voltammograms obtained for $C P E$ in $T \& K$ medium + $0.1 \mathrm{~mol} \mathrm{~L}^{-1}$ of $\mathrm{K}_{2} \mathrm{SO}_{4}$ with different chalcopyrite concentrations. $\mathrm{v}=20 \mathrm{mV}$ $s^{-1}$. (B) $Q$ versus chalcopyrite concentration plot

The charge $(\mathrm{Q})$ was measured up to the peak potential and the average values of $\mathrm{CV}$ replicates versus chalcopyrite concentration are shown in Figure 4B. A straight line with a linear coefficient near zero and a correlation coefficient, $r=0.996$ was observed. The charge measured up to the peak potential was utilized because the I-E curve after the peak is strongly dependent of chalcopyrite concentration and for low concentrations a new electrochemical process is observed, probably involving graphite.

The direct proportionality relationship between the charge and the amount of the electro-active species also suggests a homogeneous distribution of the chalcopyrite in the paste.

In order to complement the $\mathrm{CV}$ results, open-circuit potential and EIS measurements were performed. Figure 5 shows the open-circuit potential versus time curves for CPE with different chalcopyrite concentrations. The potential stabilized at shorter times and at higher values as the chalcopyrite concentration increased. Potential oscillations were mainly for the following chalcopyrite concentrations: 0 ; $2.5 ; 5$ and $10 \%$. The oscillations practically disappeared for higher mineral concentrations.

The increase in the potential as the mineral concentration increased suggests that the mixed potential is being dominated by the processes occurring on chalcopyrite instead on graphite 
surface. Probably, on graphite the main reactions were the proton reduction and the oxidation of graphite surface or its impurities. The hydrogen evolution can be responsible for the potential oscillation which diminished as the chalcopyrite concentration increased. The probable reactions occurring on the chalcopyrite surface were reduction of proton or some intermediary metallic surface and oxidation of copper (I) to copper (II) and iron (II) to iron (III) from the chalcopyrite. The changes in the nature and balance of reactions occurring at graphite and chalcopyrite surfaces explain the evolution of the open-circuit potential with the chalcopyrite concentration.
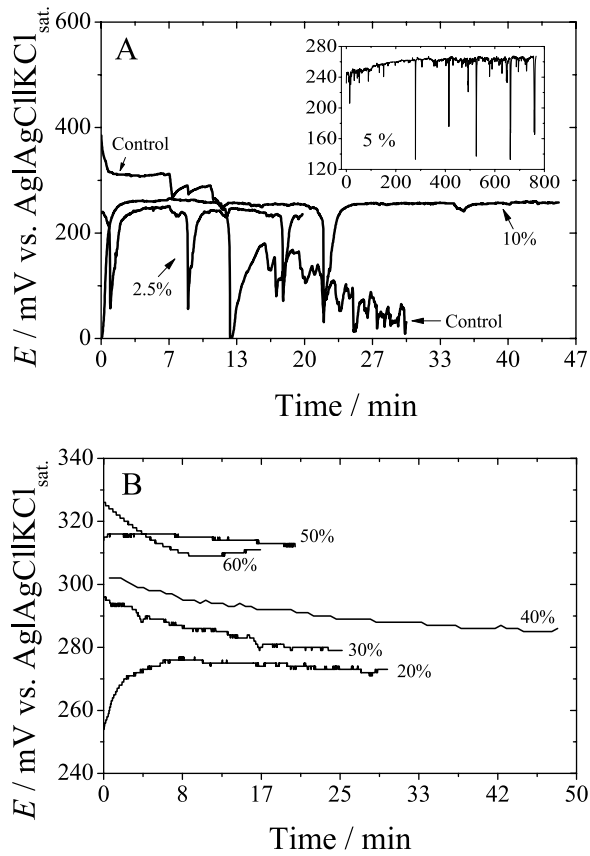

Figure 5. Open-circuit potential versus time curves obtained for CPEchalcopyrite in $T \& K$ medium, at $25{ }^{\circ} \mathrm{C}$ : (A) $0-10 \%$ of chalcopyrite; (B) $20-60 \%$ of chalcopyrite

Figure 1S (Supplementary Material) shows the impedance spectra obtained in the Bode formats for CPE modified with different chalcopyrite concentrations. A wide maximum, characteristic of non-reactive system was observed for CPEs with 0 and $10 \%$ of chalcopyrite, and some dispersion was observed at low frequency region, mainly for the paste without mineral. This dispersion may be attributed to hydrogen evolution.

At higher concentrations of chalcopyrite (20 to 60\%), two maxima in the phase angle plots were observed (Figure 1S-A). The first maximum (between 10 and $100 \mathrm{~Hz}$ ) slightly shifts to higher frequencies and smaller phase angles as the chalcopyrite concentration increased. The second time constant appeared at around 0.1 $\mathrm{Hz}$, becomes evident for chalcopyrite concentrations $\geq 20 \mathrm{wt} . \%$ and the phase angles diminished. This time constant was associated to chalcopyrite oxidation.

At high frequencies the impedance modulus (Figure 1S-B) was higher for CPE-chalcopyrite compared to the graphite electrode. For CPE-chalcopyrite a slight increase of $|\mathrm{Z}|$ was observed as the chalcopyrite concentration increased. This occurs due to the increase in the semiconductor character with mineral concentration, which increases the resistance of the electrode. At low frequencies $|\mathrm{Z}|$ values decreased about 3 orders of magnitude when the chalcopyrite concentration increased from 0 to $60 \%$ owing the increase in the quantity of the electroactive species.
Therefore, it is suggested that the first time constant was associated with processes in which the carbon surface was involved, like hydrogen evolution. The second time constant was attributed to the oxidation of the chalcopyrite. In this case different oxidation solid state reactions can be occur to form: non-stoichiometric metallic sulfides, and copper (II) and iron (III) ions as soluble species.

The CV, OCP and EIS studies indicated that CPE-(20 to $60 \mathrm{wt} . \%)$ chalcopyrite can be used to study the electrochemistry of the mineral with consistent results.

\section{CONCLUSIONS}

Comparing statistical data obtained with different pastes preparation procedures, that one kept in argon atmosphere was more reproducible since the variation in the data was significantly lower than in an air atmosphere. Therefore, some problems related with spontaneous oxidation of the pulverized chalcopyrite can be minimized by preparing it under oxygen-free atmosphere. The lowest dispersions in the charge values (Q) were observed for scan rates $(v)$ from 10 to $40 \mathrm{mV} \mathrm{s}^{-1}$. So this range can be considered appropriate to obtain reliable cyclic voltammograms of CPE-chalcopyrite electrodes.

The CPE-electrodes can be used up to around two hours after preparation for voltammetric studies of mineral sulfides dissolution with acceptable repeatability.

The open-circuit potential curves and electrochemical impedance results indicated that the response of the mineral begins to be more evident for chalcopyrite concentrations $\geq 20 \%$. Potential oscillations mainly observed for low mineral concentration were attributed to hydrogen evolution on graphite. The two time constants observed for CPE-chalcopyrite were attributed to the hydrogen evolution on graphite and chalcopyrite oxidation, respectively.

\section{SUPPLEMENTARY MATERIAL}

There is a figure in this manuscript included as supplementary material correspondent to the impedance plots (Figure 1S). This figure is freely available at http://quimicanova.sbq.org.br, as PDF file.

\section{ACKNOWLEDGMENTS}

The authors thank to CVRD (Companhia Vale do Rio Doce), CNPq (Conselho Nacional de Pesquisa e Desenvolvimento) and FAPESP (Fundação de Amparo à Pesquisa do Estado de São Paulo) for the financial support to research projects and Dr. M. V. Capela for assistance with statistical analysis of the data.

\section{REFERENCES}

1. Bevilaqua D.; Diéz-Perez, I.; Fugivara, C. S.; Sanz, F.; Benedetti, A. V.; Garcia Jr., O.; J. Braz. Chem. Soc. 2003, 14, 637.

2. Bevilaqua, D.; Diéz-Perez, I.; Fugivara, C. S.; Sanz, F.; Benedetti, A. V.; Garcia Jr., O.; Bioelectrochem. 2004, 64, 79.

3. Velásquez, P.; Leinen, D.; Pascual, J.; Ramos-Barrado, J. R.; Cordova, R.; Gómez, H.; Schrebler, R.; J. Electroanal. Chem. 2000, 494, 87.

4. Velásquez P.; Leinen, D.; Pascual, J.; Ramos-Barrado, J. R.; Cordova, R.; Gómez, H.; Schrebler, R.; J. Electroanal. Chem. 2001, 510, 20.

5. Yin, Q.; Vaughan, D. J.; England, K. E. R.; Kelsall, G. H.; Brandon, N. P.; J. Electrochem. Soc. 2000, 147, 2945.

6. Kelly, D. P.; Wood, A. P.; Int. J. Syst. Evol.Microbiol. 2000, 50, 511.

7. Rawlings, D. E.; Ann.Rev.Microbiol. 2002, 56, 65.

8. Lázaro, I.; Medina, N. M.; Rodríguez, I.; Arce, E.; González, I.; Hydrometallurgy 1995, 38, 277. 
9. Rodríguez, Y.; Ballester, A.; Blázquez, F.; González, F.; Muñoz, J. A.; Hydrometallurgy 2003, 71, 37.

10. Lu, Z. Y.; Jeffrey, M. I.; Lawson, F.; Hydrometallurgy 2000, 56, 145.

11. Toniazzo, V.; Lazaro, I.; Humbert, B.; Mustin, C.; Surf. Geosci. 1999, $328,535$.

12. Tuovinen, O. H.; Kelly, D. P.; Arch. Microbiol. 1973, 88, 285.

13. Electrochemical measurement systems hardware operators' manuals, Gamry Instruments, 2003.

14. Biegler, T.; Horne, M. D.; J. Electrochem. Soc. 1985, 132, 1363.

15. Holliday, R. I.; Richmond, W. R.; J. Electroanal. Chem. 1990, $288,83$.

16. Parker, A. J.; Paul, R. L.; Power, G. P.; J. Electroanal. Chem. 1981, 118 , 305.
17. Bevilaqua, D.; Acciari, H. A.; Arena, F. A.; Benedetti, A. V.; Fugivara, G. T. F.; Garcia Jr, O.; Miner. Eng. 2009, 22, 254.

18. Elsherief, E.; Miner. Eng. 2002, 15, 215.

19. Mikhlin, Y. L.; Tomashevich, Y. V.; Asanov, I. P.; Okotrub, A. V.; Varnek, V. A.; Vyalikh, D. V.; Appl. Surf. Sci. 2004, 225, 395.

20. Arce, E. M.; González, I.; Int. J. Miner. Process. 2002, 67, 17.

21. Bevilaqua, D.; Leite, A. L. L. C.; Garcia Jr, O.; Tuovinen, O. H.; Proc. Biochem. 2002, 38, 587.

22. Pratesi, G.; Cipriani, C.; Eur. J. Miner. 2000, 12, 397.

23. Tait, W. S.; An introduction to electrochemical corrosion testing for practicing engineers and scientists, PaisODocs Publications: Racine, 1994. 


\section{OPTIMIZATION OF THE USE OF CARBON PASTE ELECTRODES (CPE) FOR ELECTROCHEMICAL STUDY OF THE CHALCOPYRITE}

Daniela G. Horta, Denise Bevilaqua, Heloísa A. Acciari, Oswaldo Garcia Júnior* e Assis V. Benedetti Instituto de Química, Universidade Estadual Paulista, CP 355, 14801-970 Araraquara - SP, Brasil
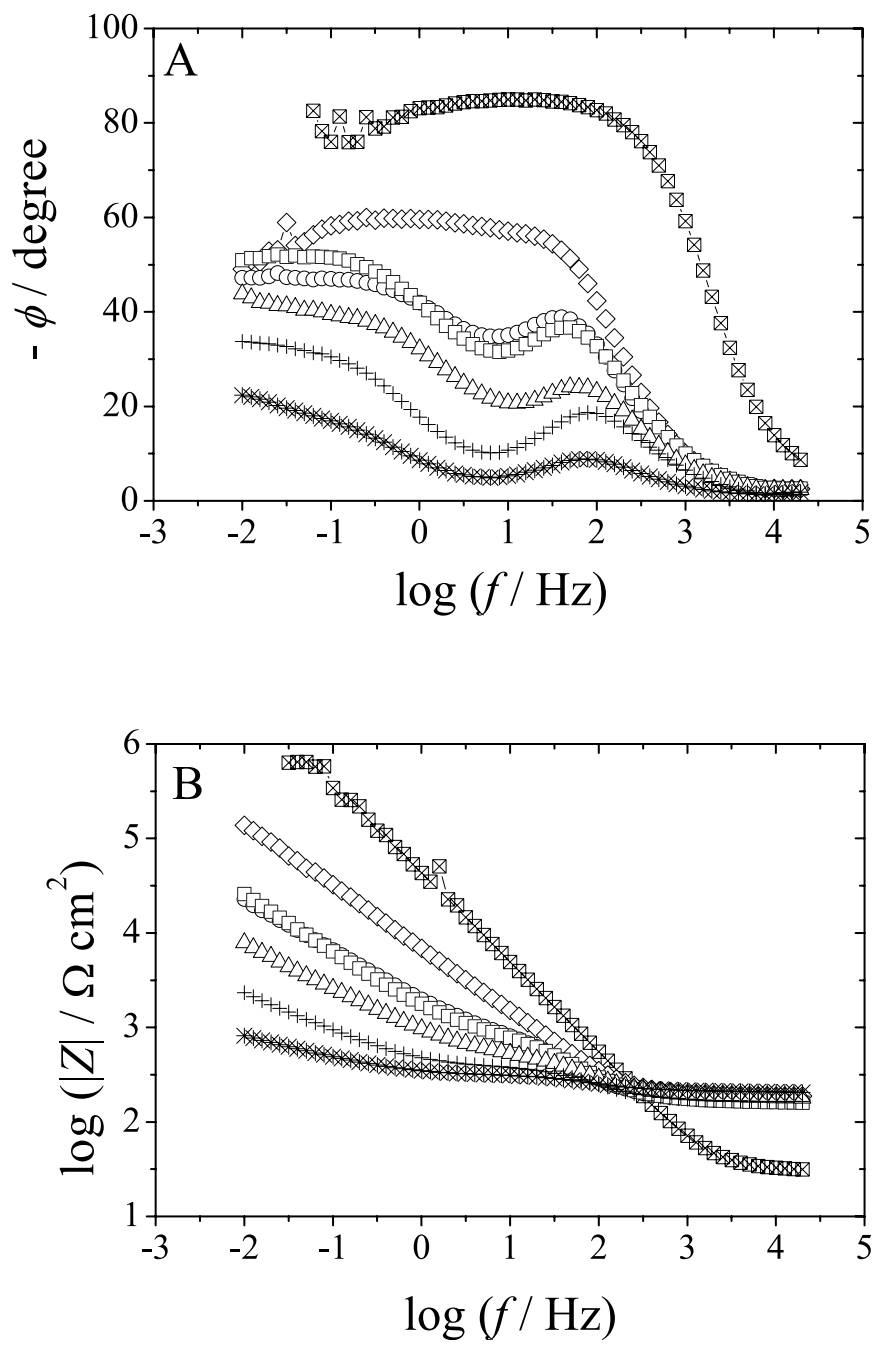

Figure 1S. Impedance spectra in the Bode format obtained for CPE-(20 wt.\%) chalcopyrite in T\&K medium: (A) phase angle (- $\phi)$ versus log (f) plots, (B) $\log (|Z|)$ versus $\log (f)$ plots. Chalcopyrite percentage in the paste: $0 \%(\bigotimes), 10 \%(\diamond), 20 \%(\bigcirc), 30(\square), 40 \%(\triangle), 50 \%(+), 60 \%(*)$ 